\section{Mikronährstoff - Forschung an den agrarwissenschaftlichen Instituten in Rostock - ein Rückblick}

\author{
Werner Borchmann \\ Bremer Str. 2 \\ D - 18057 Rostock
}

\section{ZUSAMMENFASSUNG}

Ergebnisse der Mikronährstoff - Forschung an den Rostocker agrarwissenschaftlichen Instituten werden mitgeteilt.

Entscheidungskriterien für eine ökologisch und ökonomisch vertretbare Mikronährstoffanwendung als Boden- und Blattdünger werden diskutiert.

Schlüsselworte: Mikronährstoffe, Düngungsbedarf, Applikationsverfahren, Terminierung, Blattdüngung

In den letzten Jahren sind unsere Kenntnisse über die umweltschonende Nährstoffversorgung ertragsund qualtitätsorientierter Pflanzenbestände bedeutsam erweitert worden. Ökologische und ökonomische Sachzwänge sind in den Vordergrund praktischer Überlegungen zur Düngeranwendung getreten. Neben den Makronährstoffen spielen die zu den Mikronährstoffen bzw. Spurennährstoffen zählenden Nährelemente eine zunehmend wichtige Rolle. Von den Pflanzen nur in relativ geringen Mengen erforderlich sind:

- Kupfer, Mangan, Zink, Molybdän, Eisen

- Bor, Chlorid.

In der Tier- und Humanernährung sind Bor und Molybdän nicht bzw. umstritten lebenswichtig, dafür aber neben weiteren Kobalt, Jod, Fluor und Selen.

Untersuchungen zum Gehalt und zur Dynamik von Mikronährstoffen bzw. Spurenelementen im System Boden - Pflanze - Tier haben an den Rostocker agrarwissenschaftlichen Instituten eine langjährige Tradition. Bereits im Jahre 1942 wurden vom damaligen Direktor der Landwirtschaftlichen Untersuchungs- und Forschungsanstalt (LUFA) Prof. Dr. Dr. h. c. mult. K. Nehring ein Kupferdüngungs Feldversuch angelegt.

K. Nehring war gleichzeitig Direktor des Institutes für Agrikulturchemie und Bodenkunde an der Landwirtschaftlichen Fakultät der Universität Rostock. Die Mitarbeiter beider Einrichtungen, die auch meine Arbeitsstätten waren, haben diese Arbeitsrichtung aufgegriffen und mit erweiterten Zielstellungen erfolgreich betrieben.

Die ursprüngliche Aufgabe der Rostocker Arbeiten resultierte aus aktuellen Fragestellungen zur gesunden Tierernährung mit vorwiegend wirtschaftseigenem Futter. Nach einer Konzeption von Prof. Nehring sollten im Rahmen einer „Heuwertprüfung“ von den fünf Landwirtschaftlichen Versuchsstationen (Rostock, Halle, Leipzig, Jena und Potsdam) der ehemaligen DDR u.a. Aussagen über den durchschnittlichen Mineralstoffgehalt in Wiesenheu erarbeitet werden. Für das Rostocker Einzugsgebiet waren dabei auch die für die Tierernährung relevanten und möglicherweise im Mangelbereich liegenden Mangan-, Kupfer- und Kobaltgehalte zu ermitteln.

Im Rahmen dieser Heuwertprüfung wurden in Gemeinschaftsarbeit der Mikronährstofflaboratorien der Landwirtschaftlichen Untersuchungs- und Forschungsanstalt (LUFA) und des Institutes für Agrikulturchemie und Bodenkunde der Landwirtschaftlichen Fakultät Rostock rund 2.600 Wiesenheuproben aus dem Territorium Mecklenburg-Vorpommern auf den Gehalt an Mangan, Kupfer und Kobalt untersucht und bewertet (Nehring u. Borchmann, 1959, 1961; Borchmann, 1964; Nehring, 1965).

Gleichzeitig wurden Mikronährstoff Bestimmungen in zahlreichen Böden durchgeführt.

Typische Kupfer- und Mangan Mangelstandorte, besonders auf Niedermoor- und Sandböden, konnten ausgewiesen werden.

Die zusammengefassten Ergebnisse der in der ehemaligen DDR durchgeführten Mikronährstoff Bodenuntersuchungsergebnisse sind in Tabelle 1 dargestellt.

Tabelle 1

Stand der Mikronährstoffversorgung der Böden der ehemaligen DDR - Untersuchungszeitraum 1984 - 1988 (Witter, 1990)

\begin{tabular}{|l|r|r|r|r|}
\hline \multirow{2}{*}{ Element } & \multirow{2}{*}{$\begin{array}{c}\text { Erfasste } \\
\text { Fläche }\end{array}$} & \multicolumn{3}{|c|}{ Prozentualer Flächenanteil } \\
\cline { 3 - 5 } & In Tha & \multicolumn{1}{c|}{$\begin{array}{c}\text { hoher } \\
\text { Gehalt }\end{array}$} & $\begin{array}{c}\text { mittlerer } \\
\text { Gehalt }\end{array}$ & $\begin{array}{c}\text { niedriger } \\
\text { Gehalt }\end{array}$ \\
\hline $\mathrm{B}$ & 2234 & 58 & 26 & 16 \\
\hline $\mathrm{Cu}$ & 2709 & 20 & 71 & 9 \\
\hline $\mathrm{Mn}$ & 904 & 89 & 9 & 2 \\
\hline $\mathrm{Mo}$ & 704 & 57 & 36 & 7 \\
\hline $\mathrm{Zn}$ & 394 & 61 & 36 & 3 \\
\hline
\end{tabular}

Aus diesen bereits seit 1960 laufenden nahezu flächendeckenden Untersuchungen wurden praxisrelevante Empfehlungen zur gezielten Mikronährstoffdüngung und / oder Empfehlungen zur Verwendung von Mikronährstoffzusätzen in der Tierernährung abgeleitet.

Die Notwendigkeit tierphysiologisch bedeutsamer Mikronährstoffgehalte z.B. des Kupferanteiles im Grünfutter von Hochleistungskühen wird in der Literatur erneut diskutiert. Bergmann u. Beringer (2001) vermuten bei unzureichendem Kupferangebot in Verbindung mit gestörter Kupferverwertung einen Faktor, der das Auftreten von BSE-Erkrankungen bei Rindern begünstigen kann. 


\section{MIKRONÄHRSTOFFBEDARF}

Auf den Standorten mit traditioneller Pflanzenproduktion ist die Eisenversorgung der Pflanzenbestände nur in seltenen Fällen gestört Eine Unterversorgung - z.T. durch $\mathrm{zu}$ hohe, auch $\mathrm{zu}$ niedrige Boden - $\mathrm{pH}$-Werte bedingt und gelegentlich im Garten-, Zierpflanzen- und Weinbau vorkommend, kann durch Chelate, z.B. Fetrilon $\left(\begin{array}{llll}13 \% & \mathrm{Fe}\end{array}\right)$ oder Folicin DP $(6 \%$ Fe $)$ wirksam aufgehoben werden.

Eine zusätzliche Chloridversorgung spielt gegenwärtig keine Rolle. Neben der beständigen Nachlieferung aus dem natürlichen Bodenmaterial ist eine kontinuierliche Chloridzufuhr durch die Anwendung chloridhaltiger Kalidüngersalze gegeben. Dieser zusätzliche Chloridschub kann auf dafür prädestinierten Standorten und in exponierten Klimalagen - Kontinentalklima - ertrags- und qualitätsstabilisierend auf salz- bzw. chloridliebenden Pflanzen, wie z.B. Betarüben, Kohlgewächse u.a. wirken.

Im Vergleich zu den Mikronährstoffen Eisen und Chlor ist der Stellenwert von Bor, Kupfer, Mangan, Zink und Molybdän bedeutsam höher. Gründe hierfür sind die auf chemisch analytischem Wege (Boden-/ Pflanzenanalyse) bzw. visuellem Wege (akute Mangelerscheinungen) festgestellten Mikronährstoffdefizite in Böden und / oder Pflanzen, die im Regelfall mit Ertrags-, Qualitäts- und auch Resistenzminderungen verbunden sind. Die mittleren Mikronährstoffentzüge im Rahmen der Fruchtfolge werden in Tabelle 2 angegeben.

Tabelle 2

Mittlere Mikronährstoffentzüge und empfohlene Düngergaben

\begin{tabular}{|l|r|r|r|}
\hline Nährstoff & \multicolumn{1}{|c|}{$\begin{array}{c}\text { Entzug } \\
\text { g/ha }\end{array}$} & $\begin{array}{c}\text { Empfohlene } \\
\text { Bodendüngung } \\
\text { kg/ha }\end{array}$ & $\begin{array}{c}\text { Blattdüngung } \\
\text { g/ha }\end{array}$ \\
\hline Bor & $120-500$ & $1-2$ & 400 \\
\hline Mangan & $300-1000$ & - & 1000 \\
\hline Kupfer & $50-100$ & $2-5$ & 500 \\
\hline Molybdän & $4-20$ & 0,5 & 300 \\
\hline Zink & $200-300$ & -5 & 500 \\
\hline
\end{tabular}

Mikronährstoffentzug und -bedarf (Düngungsbedarf) sind nicht identische Größen. Im Vergleich zu den Makronährstoffen liegt die Höhe der entzugsdeckenden Mikronährstoffgaben insbesondere bei Bodendüngung - zwischen dem 5 100 und mehrfachen des Entzuges. Beide Tendenzen

- unzureichende Verwertung der NPKDüngergaben bei Mikronährstoffmangel oder

- unkontrollierte, teilweise $\mathrm{zu}$ hohe, sog. Vorbeugende Mikronährstoffgaben

belasten das Ökosystem und sind stets mit Resistenz-, Ertrags- und Qualitätsminderungen verbunden. Hierin liegen die Ansatzpunkte zur Entwicklung praxisrelevanter Mikronährstoffdüngungsverfahren.
KONZEPT DER ÖKOLOGISCH ORIENTIERTEN MIKRONÄHRSTOFFDÜNGUNG

Zur Verhinderung ökologischer und ökonomischer Fehlleistungen durch eine unterlassene bzw. überhöhte Mikronährstoffdüngung sind von uns im Rahmen des ehemaligen Arbeitskreises „Mikronährstoffdüngung der Akademie der Landwirtschaftswissenschaften“, dessen langjähriger Leiter Prof. Bergmann war, Konzepte entwickelt und Empfehlungen erarbeitet worden.

\section{MIKRONÄHRSTOFF - BODENDÜNGUNG}

Eine ökologisch und ökonomisch vertretbare Bodendüngung setzt die Kenntnis des pflanzenverfügbaren Mikronährstoff - Ist - Gehaltes eines Feldschlages voraus. Die Nährstoffgehalte der Böden werden von den zuständigen Landwirtschaftlichen Untersuchungs- und Forschungsanstalten (LUFA) der Länder ermittelt. Die Gehalte des jeweiligen Elementes werden an Hand von Grenzwerttabellen und unter Berücksichtigung wichtiger, die Mikronährstoffaufnahme beeinflussender Faktoren, wie z.B. Anteil organischer Bodensubstanz, pHWert, Feinanteil u.a. Parameter drei Gehaltsklassen $(A-C-E)$ zugeordnet. Dabei entspricht:

Gehaltsklasse A: niedriger Bodennährstoffgehalt Düngung erforderlich

Gehaltsklasse C: anzustrebender optimaler Bodennährstoffgehalt

Gehaltsklasse E: sehr hoher Bodennährstoffgehalt Düngung nicht erforderlich

Für unser Land Mecklenburg-Vorpommern (MV) werden von der Landwirtschaftlichen Versuchsund Forschungsanstalt Rostock folgende Ergebnisse mitgeteilt:

\section{Mikronährstoffversorgung des Ackerlandes von M-V Ergebnisse des Jahres 1997 in \% Flächenanteil (Schweder, 1998)}

\begin{tabular}{|l|r|r|r|r|}
\hline \multirow{2}{*}{$\begin{array}{c}\text { Mikronäh } \\
\text { rstoff }\end{array}$} & Anzahl & \multicolumn{3}{|c|}{ Gehaltsklassen } \\
\cline { 3 - 5 } & Proben & \multicolumn{1}{|c|}{ A } & \multicolumn{1}{c|}{ C } & \multicolumn{1}{c|}{ E } \\
\hline Bor & 2385 & 25,9 & 46,8 & 27,3 \\
\hline Kupfer & 1853 & 2,6 & 70,2 & 27,2 \\
\hline
\end{tabular}

Die Ergebnisse der Bodenuntersuchung bilden die Grundlage für die unter Berücksichtigung weiterer boden - /pflanzenspezifischer Parameter auf der Grundlage entsprechender Computerprogramme berechneten Düngungsempfehlungen durch die zuständige LUFA. Den Landwirtschafts- bzw. Gartenbaubetrieben stehen bewährte Produkte in Form von vorwiegend Mehrnährstoff-, aber auch Einzelnährstoffdüngern zur Verfügung. In diesem Zusammenhang soll darauf hingewiesen werden, dass Dünger organischen Ursprungs - insbesondere Stalldung, Gülle, Kompost u.a. zur Mikronährstoffversorgung der Böden beitragen. Zu 
beachten ist, dass sämtliche, als mikronährstoffhaltig angebotene organische bzw. mineralische Produkte den Forderungen des Düngemittelrechtes entsprechen müssen.

\section{MIKRONÄHRSTOFF - BLATTDÜNGUNG}

Die Blattdüngung stellte ursprünglich ein Verfahren zur Beseitigung beginnender visuell erkennbarer Nährstoffmangelerscheinungen an wachsenden Pflanzenbeständen dar. Mit den zunehmenden chemisch analytischen Möglichkeiten wurde dieses Konzept erweitert. Wir sehen in der Blattdüngung nicht nur ein Reparaturverfahren zur Beseitigung von Nährstoffmangel während der Vegetationsperiode, sondern eine aus Diagnoseverfahren abgeleitete gezielte Nährstoffzufuhr zur Ausschöpfung des genetisch bedingten Ertrags- und Qualitätspotentials. Da die Blattdüngung ein „Behandlungsverfahren“ in wachsenden Pflanzenbeständen ist, sind die ökologischen Anforderungs- und Entscheidungskriterien besonders hoch anzusetzen. In gebündelter Form sind diese in Abbildung 1 zusammengefasst.

Abbildung 1: Entscheidungskriterien einer ökologisch orientierten Blattdüngung

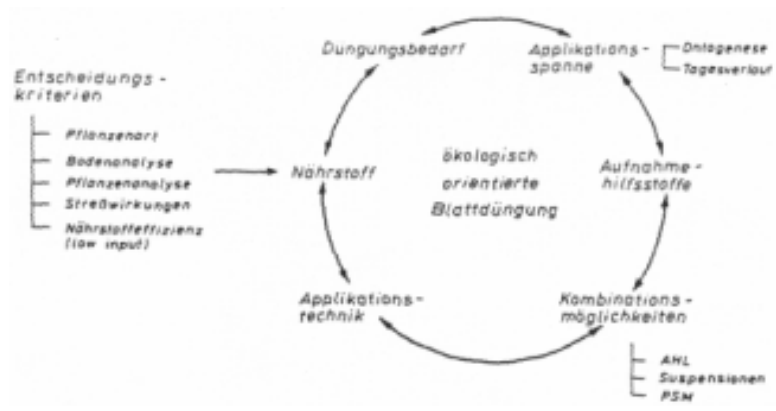

Bezüglich der Ansprüche einiger ausgewählter Pflanzenarten an die Mikronährstoffversorgung, und das ist auch ein Entscheidungskriterium für die gegebenenfalls erforderliche Bodendüngung, bestehen folgende Unterschiede:

\begin{tabular}{|l|l|}
\hline Relativ hoher Anspruch & Kultur \\
\hline $\mathrm{B}$ & $\begin{array}{l}\text { Raps, Rüben, Leguminosen, } \\
\text { Kohlarten }\end{array}$ \\
\hline $\mathrm{Cu}$ & $\begin{array}{l}\text { Weizen, Gerste, Hafer, Grünland, } \\
\text { Sonnenblumen }\end{array}$ \\
\hline $\mathrm{Mn}$ & $\begin{array}{l}\text { Weizen, Gerste, Hafer, Raps, Rüben, } \\
\text { Erbsen }\end{array}$ \\
\hline $\mathrm{Mo}$ & $\begin{array}{l}\text { Kleinkörnige Leguminosen, Raps, } \\
\text { Futterkohl }\end{array}$ \\
\hline $\mathrm{Zn}$ & Mais, Gemüse \\
\hline
\end{tabular}

Neben den längerfristig orientierenden Ergebnissen der Mikronährstoff - Bodenanalyse geben die Werte der Pflanzenanalyse den aktuellen Stand der Nährstoffversorgung des Pflanzenbestandes wieder. An Hand von
Grenzwerttabellen können Schlussfolgerungen für die gegebenenfalls erforderliche bzw. nicht notwendige Blattdüngung gezogen werden. Zur praktischen Durchführung des Pflanzenanalyseverfahrens - wie Probenahmetermin, Pflanzenorgan, Angaben zur Düngermenge u.a.m. erteilen die LUFA präzisierte Angaben. Bei der Ermittlung des Düngerbedarfes zur Blattapplikation sind nicht nur pflanzenartenabhängige Mikronährstoffansprüche zu beachten. Zunehmende Bedeutung hat die Nährstoffeffizienz einzelner Sorten.

In einigen, an unserem Institut durchgeführten Arbeiten (Borchmann u. Zajonc, 1988; Elscheich, 1990; Pöplau u. Zajonc, 1991) konnten wir feststellen, dass die sortenspezifischen Reaktionen im Mikronährstoff - Verwertungsvermögen teilweise größer sind als die artenspezifischen. Teilergebnisse der Prüfung von Winterweizensorten hinsichtlich ihrer $\mathrm{Cu}-$ bzw. Mn-Effizienz sind in Tabelle 3 angeführt.

Tabelle 3

Mikronährstoffeffizienz verschiedener Winterweizensorten

\begin{tabular}{|l|r|r|}
\hline \multirow{2}{*}{ Winterweizensorte } & \multicolumn{2}{|c|}{$\begin{array}{c}\text { Prozentualer Ertragsausfall bei } \\
\text { unzureichender }\end{array}$} \\
\cline { 2 - 3 } & Cu-Versorgung & Mn-Versorgung \\
\hline Taras & 72 & 19 \\
\hline Compal & 30 & 46 \\
\hline
\end{tabular}

Ersichtlich ist, dass z.B. die Sorte „Taras“ auf eine $\mathrm{Cu}$-Unterversorgung des Bodens mit einem höheren Ertragsausfall reagiert, als „Compal“. Entgegengesetzt dazu reagiert die Sorte „Compal“ empfindlicher auf eine Mn-Unterversorgung als Bestände der Sorte „Taras“. Die Fähigkeit einer Pflanzensorte, boden- bzw. blattapplizierte Nährstoffe besser zu verwerten und in Ertrags- und Qualitätsleistungen umzusetzen, hat verschiedene Ursachen - differenzierte Wurzelentwicklung, begrenztes Nährstoff-aufschlußvermögen, Nährstofftransport - $\quad$ bzw. Verlagerungsbesonderheiten während der Ontogenese u.a.m. werden diskutiert. Die zur erhöhten Nährstoffeffizienz führenden Eigenschaften sind z.T. genetisch fixiert. Durch Gentransfer wird im Interesse der besseren Nährstoffverwertung und einsparung an der Züchtung von low input Sorten gearbeitet (Gerath, 1998).

\section{APPLIKATIONSZEITPUNKT}

Der Erfolg einer Blattdüngung ist neben den genannten Kriterien weitgehend vom Applikationszeitpunkt abhängig. Nach mehrjähriger Versuchsarbeit unterbreiteten wir Konzepte der terminierten Mikronährstoffdüngung. In ihnen präzisierten und empfahlen wir physiologisch begründete und technologisch realisierbare Zeitspannen der Mn- und z.T. Cu-Düngung im Getreidebau sowie für die B- und Mn-Düngung zu Winterraps (Engelke, 1987; Röhl, 1988; Zajonc et al., 
1985, Borchmann et al., 1988). In vereinfachter Form sind in Abbildung 2 die empfohlenen Zeitspannen für eine Mn-Blattdüngung $\mathrm{zu}$ einigen Getreidearten dargestellt.

Abbildung 2: Empfohlene Zeitspannen für eine MnBlattdüngung (nach Zajonc)

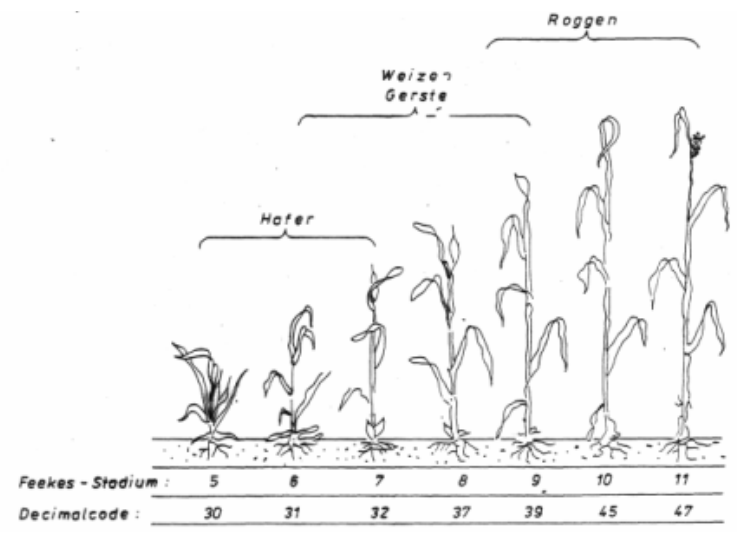

Bei Nichteinhaltung der pflanzenphysiologisch begründeten Anwendungszeitspannen ist der kalkulierte Ertrags- / Qualitätserfolg der Blattdüngung in Frage gestellt. Entsprechende Auswirkungen sind aus Tabelle 4 ableitbar.

Tabelle 4

Ertragswirksamkeit einer terminierten Mikronährstoffdüngung bei Winterraps (Gefäßversuche)

\begin{tabular}{|l|r|r|}
\hline \multirow{2}{*}{$\begin{array}{c}\text { Applikationszeitpunkt } \\
\text { zum Entwicklungsstadium }\end{array}$} & \multicolumn{2}{|c|}{$\begin{array}{c}\text { Rel. Kornertrag bei } \\
\text { Blattdüngung } \\
\text { (ohne Mikronährstoffe = 100) }\end{array}$} \\
\cline { 2 - 3 } & Mn & B \\
\hline $\begin{array}{l}\text { Rosettenstadium / } \\
\text { Knospenstadium - klein }\end{array}$ & 100 & 102 \\
\hline Knospenstadium - mittel & $\mathbf{1 0 9}$ & 98 \\
\hline Knospenstadium - groß & $\mathbf{1 1 9}$ & 99 \\
\hline Blühbeginn & 102 & $\mathbf{1 1 8}$ \\
\hline Vollblüte & 96 & $\mathbf{1 1 1}$ \\
\hline
\end{tabular}

\section{AUFNAHMEHILFSSTOFFE}

Blattdünger, makro- und / oder mikronährstoffhaltige, werden in flüssiger Form vorwiegend als Tröpfchen unterschiedlicher Größe auf die Blattoberfläche gebracht. Tropfenform und Wachsschicht - insbesondere bei Dicotyledonen hindern die schnelle Passage des Pflanzennährstoffes zum Cytoplasma. Zur Abschwächung dieser Effekte wurden zahlreiche, zur Gruppe der Tenside zählende Aufnahmehilfsstoffe entwickelt. Auf Grund ihrer grenzflächenaktiven Eigenschaften - Erniedrigung der Grenzflächenspannung des Wassertropfens steigern Tenside die Verfügbarkeit applizierter Pflanzennährstoffe, in dem sie eine Verbesserung der Benetzung der Blattoberfläche bewirken bzw. die Durchlässigkeit der Cuticula erhöhen. Im Rahmen einer Promotionsarbeit entwickelte Koziolek (1992) an unserem Institut mehrere Zuckertenside und testete deren Wirksamkeit bzgl. der Effektivität einer Bordüngung bei Raps (Tabelle 5).

Tabelle 5

Einfluß von Zuckertensidzusätzen zur Bor - Blattdüngung bei Raps (Koziolek, 1992)

\begin{tabular}{|l|r|r|}
\hline \multicolumn{1}{|c|}{ B-Blattdüngung } & \multicolumn{1}{|c|}{$\begin{array}{c}\text { B-Gehalt } \\
\text { Hg / gTM }\end{array}$} & \multicolumn{1}{c|}{$\begin{array}{c}\text { Aufnahmever- } \\
\text { besserung }\end{array}$} \\
\hline B-Lösung & 46 & 100 \\
\hline $\begin{array}{l}\text { B-Lösung + Zuckertensid } \\
\text { (hydrophil) }\end{array}$ & 68 & 147 \\
\hline $\begin{array}{l}\text { B-Lösung + Zuckertensid } \\
\text { (lipophil) }\end{array}$ & 59 & 128 \\
\hline
\end{tabular}

Durch Zuckertenside wurde die B-Aufnahme nach Blattdüngung bedeutsam erhöht. Die Wirkung von Tensiden mit hydrophilem bzw. lipophilem Charakter war unterschiedlich. Als sehr wirksamer Aufnahmehilfsstoff erwies sich das Zuckertensid 4.6 - o - Alkaliden - D - Glycopyronosen. Der Entwicklung biologisch leicht abbaubarer Tenside kommt zukünftig bei der Blattdüngung aber auch bei der Anwendung von Pflanzenschutzmitteln größere Bedeutung zu.

Die Leistungsfähigkeit der Mikronährstoff Forschung in Rostock spiegelte sich in zahlreichen Publikationen, Forschungsberichten und in der Mitgestaltung wissenschaftlicher nationaler und internationaler Tagungen wider. Einer besonderen Würdigung bei diesem Rückblick bedarf die erfolgreiche Zusammenarbeit mit dem Lehrstuhl Agrikulturchemie und ihrem Leiter - Herr Prof. Dr. Dr. h.c. J. Loch - an der Agraruniversität Debrecen / Ungarn.

Am Lehrstuhl Pflanzenernährung der Agrarwissenschaftlichen Fakultät wurden angefertigt: 75 Forschungsberichte (unveröffentlicht), 5 Habilitationsschriften, 12 Promotionsschriften und 86 Diplomarbeiten, die sich ausschließlich mit ernährungs- und düngungsspezifischen Wirkungen von Mikronährstoffgaben $\mathrm{zu}$ verschiedenen Pflanzenarten und -sorten befassten.

Genannt werden sollen auch die zahlreichen fotografischen Aufnahmen von Mikronährstoff Mangelerscheinungen an landwirtschaftlichen und gärtnerischen Kulturen durch Mitarbeiter beider Mikronährstoff - Laboratorien. Viele Bilddokumente sind in dem einmaligen Standardwerk von Bergmann (1988) verarbeitet.

Der bisherige Umfang der von der LUFA Rostock durchgeführten Mikronährstoffuntersuchungen in Böden und Pflanzen gestattet eine flächendeckende Beurteilung des Mikronährstoff - Status der wichtigsten Ackerund Grünland - Standorte MecklenburgVorpommerns. Mikronährstoffuntersuchungen in Böden, Pflanzen und düngungsrelevanten Produkten werden von den Landwirtschaftsbetrieben wieder zunehmend gewünscht.

Demgegenüber kam die praxisorientierte, pflanzenphysiologisch - experimentell begründete Mikronährstoff - Forschung an der Universität nach 
der politischen Wende weitgehend zum Erliegen (Fibian, 1998). Strukturveränderungen führten an der Agrarwissenschaftlichen Fakultät der Universität Rostock im Januar 1992 zur Abwicklung des Lehrstuhles Pflanzenernährung / Düngung. Die langjährige universitäre Mikronährstoff - Forschung wurde eingestellt.

Im März 2002 erfolgte im Fachbereich Agrarökologie der Fakultät für Agrar- und
Umweltwissenschaften die Gründung des Institutes für Bodenkunde und Pflanzenernährung - Direktor Prof. Dr. Leinweber. Nach 10-jähriger Arbeitseinschränkung ist zu erwarten, dass sich das Lehr- und Forschungsgebiet Pflanzenernährung mit neuen Erkenntnissen zur umweltgerechten Landbewirtschaftung weiterentwickelt (Köppen u. Loch, 1998).

\section{LITERATUR}

Bergmann, W. (1988): Ernährungsstörungen bei Kulturpflanzen. Gustav Fischer Verlag Jena, $762 \mathrm{~S}$.

Bergmann, W. - Beringer, H. (2001): Kupfermangel, ein möglicher BSE-auslösender Faktor? Plant Nutr. Soil Sci, 164, 233-235.

Borchmann, W. (1964): Untersuchungen über den Mangan-, Kupfer- und Kobaltgehalt landwirtschaftlicher Futterpflanzen und dessen Beeinflussung durch exogene Faktoren. Habil.Schrift Landw. Fak., Universität Rostock, 250 S.

Borchmann, W. - Zajonc, I. - Röhl, W. (1988): Stand und Perspektiven der Blattdüngung. Rostocker Agrarwiss. Beiträge, Universität Rostock, H. 20, 13-23.

Borchmann, W. - Zajonc, I. (1998): Pflanzenernährung Entwicklung von Lehre und Forschung an der Agrarwissenschaftlichen Fakultät Rostock, EhrenkolloquiumAgrarwiss. Fak. Rostock, 13-24.

Elscheich, A. N. (1990): Aufnahme und Verwertung von Kupfer und Mangan durch verschiedene Weizensorten. Diss. Agrarwiss. Fak. Universität Rostock, 102 S.

Engelke, G. (1987): Untersuchungen zur Cu-Düngung bei Getreide unter Berücksichtigung der Terminierung von CuBlattapplikationen. Diss. Agrarwiss. Fak. Universität Rostock

Fibian, K. D. (1998): Eignung von Weiden zur Phytoremediation schwermetallbelasteter Böden und Substrate. Ehrenkolloquium, Agrarwiss. Fak. Universität Rostock, 5566.

Gerath, H. (1998): Möglichkeiten zur Steigerung der StickstoffEffizienz bei Winterraps. Ehrenkolloquium, Agrarwiss. Fak. Universität Rostock, 45-53.

Köppen, D. - Loch, J. (1998): Aspekte der umweltgerechten Landbewirtschaftung. Ehrenkolloquium, Agrarwiss. Fak. Universität Rostock, 25-35.
Koziolek, D. (1992): Eignung von Kohlenhydrattensiden als Additive bei der Bor-Blattapplikation zu Raps. Diss. Agrarwiss. Fak. Universität Rostock, 145 S.

Nehring, K. - Borchmann, W. (1959): Mikronährstoffgehalte im Heu mecklenburgischer Wiesen. Zeitschrift für Landw. Versuchs- und Untersuchungswesen. Band 5, 556-570.

Nehring, K. - Borchmann, W. (1961): Die Bedeutung der Mikronährstoffe auf dem Grünland. Dt. Landw., 12, 538-541.

Nehring, K. (1965): Düngung, Qualität und Futterwert. Handbuch der Pflanzenernährung und Düngung. Springer Verlag Wien, 1261-1354.

Pöplau, R. - Zajonc, I. (1991): Untersuchungen zum MnVerwertungsvermögen von zwei Wintergerstensorten. Mengen- und Spurenelemente, 11. Arbeitstagung, Leipzig, 164-171.

Röhl, W. (1988): Terminierung der Bor-Mangan-Blattdüngung zu Winterraps mit Einfach- und Doppelqualität unter Berücksichtigung der Ertragskomponenten und Qualitätsparameter. Diss. Agrarwiss. Fak. Universität Rostock.

Schweder, P. (1998): Nährstoffversorgung der Ackerlandes in Mecklenburg-Vorpommern. Ehrenkolloquium, Agrawiss. Fak. Universität Rostock, 34-44.

Witter, B. (1990): Die Mikronährstoffversorgung des Ackerlandes. - 30 Jahre Mikronährstoff- und Spurenelementforschung in Jena. 6, 22-27.

Zajonc, I. - Borchmann, W. - Röhl, W. (1985): Die Wirkung einer terminierten Bordüngung auf die Ertragskomponenten von Sommerraps. - Mengen- und Spurenelemente. Arbeitstagung Leipzig, 148-154. 